\title{
Does a slight change in serum creatinine matter in coronavirus disease 2019 (COVID-19) patients?
}

\author{
Yohei Komaru $^{1}$, Kent Doi ${ }^{2}$ \\ ${ }^{1}$ Department of Hemodialysis and Apheresis, The University of Tokyo Hospital, Tokyo, Japan \\ ${ }^{2}$ Department of Acute Medicine, The University of Tokyo Hospital, Tokyo, Japan
}

\section{See Article on Page 231-240}

Acute kidney injury (AKI) is one of the most frequent complications in patients with severe acute respiratory syndrome coronavirus 2 (SARS-CoV-2) infection during the coronavirus disease 2019 (COVID-19) pandemic. Although COVID-19 is characterized by atypical pneumonia and subsequent severe respiratory failure, approximately $10 \%$ of inpatients with COVID-19 reportedly suffer from AKI [1], which is significantly associated with poor outcomes [2]. Successive recent publications have reported an even higher global incidence of AKI. Patients with COVID-19 who are being treated in intensive care units (ICUs) are the most susceptible to severe AKI, which requires renal replacement therapy. However, the pathophysiology of COVID-19-associated AKI remains to be determined; systemic inflammation, hypovolemia, nephrotoxic drugs, various lung-kidney interactions (such as possible renal congestion presumably caused by high levels of positive end expiratory pressure), and direct viral cytopathic effects on the renal epithelium and podocytes are all possible mechanisms currently in discussion [3]. With no specific treatment for COVID-19-asso- ciated AKI available, early recognition of patients at risk and early diagnosis of AKI are key to confronting this emerging health burden on clinical nephrology.

In this issue of Kidney Research and Clinical Practice, Alfano et al. [4] reported that absolute change in serum creatinine level $(\Delta \mathrm{sCr})$ within 24 hours of admission was significantly associated with 30-day in-hospital mortality in patients with COVID-19. The authors conducted a retrospective observational study on 224 non-ICU inpatients at a single center in Italy and stratified them into three groups: 1) stable kidney function group with $\Delta \mathrm{sCr}$ within the range of $-0.05 \mathrm{mg} / \mathrm{dL}$ to $+0.05 \mathrm{mg} / \mathrm{dL}, 2)$ decreased kidney function group with $\Delta \mathrm{sCr}$ higher than $+0.05 \mathrm{mg} / \mathrm{dL}$, and 3) improved kidney function group with $\Delta \mathrm{sCr}$ lower than $-0.05 \mathrm{mg} / \mathrm{dL}$. They reported the highest survival rate in the stable kidney function group, while the decreased kidney function group demonstrated the highest rates of both AKI and mortality during hospitalization.

The study by Alfano et al. [4] offers evidence that even modest renal dysfunction during the early stage of admission is associated with poor prognosis in patients with COVID-19 who are treated in general wards. The timing of AKI development in SARS-CoV-2 infection must be considered. In

Received: May 13, 2021; Revised: May 13, 2021; Accepted: May 14, 2021

Editor: Tae-Hyun Yoo, Yonsei University, Seoul, Republic of Korea

Correspondence: Kent Doi

Department of Acute Medicine, The University of Tokyo Hospital, 7-3-1 Hongo, Bunkyo-ku, Tokyo 1138655, Japan. E-mail: kdoi-tky@umin.ac.jp ORCID: https://orcid.org/0000-0002-0115-1439 
previous investigations of COVID-19, two phenotypes of AKI were reported: 1) AKI that was already present at the time of admission and 2) AKI that newly developed about one week after admission [2,5]. According to Hirsch and colleagues' report on 5,449 patients hospitalized with COVID-19 in New York [2], 37.3\% of patients with AKI either arrived with AKI or developed it within 24 hours of admission. The current report by Alfano et al. [4] calls attention to the former group that had evident renal dysfunction during the early stage of admission. Further evaluation is necessary to clarify the impact of newly developed AKI during hospitalization on patient outcomes; Chan et al. [6] reported that about $35 \%$ of COVID-19 patients who experienced an AKI episode during hospitalization had residual renal impairment even after discharge.

Another point highlighted by Alfano et al. [4] is that the threshold for deteriorating or improving renal function was much smaller in their patients than described in previous reports $(0.05 \mathrm{mg} / \mathrm{dL}$ in serum creatinine). This very slight change in serum creatinine is likely to be recognized as nonsignificant in clinical practice. However, the risk stratification associated with this threshold showed a significant difference in survival of patients with COVID-19. The Acute Kidney Injury Network first incorporated an absolute increase of $0.3 \mathrm{mg} / \mathrm{dL}$ in serum creatinine into the AKI diagnostic criteria, which is valid in its present form as revised by the Kidney Disease Improving Global Outcomes. The rationale behind these international guidelines included several preceding studies on patients after cardiac surgery [7] and in general inpatient settings [8]. In the present study by Alfano et al. [4], even in the decreased kidney function group, only $30 \%$ of the COVID-19 patients met the current AKI diagnostic criteria. The current threshold of serum creatinine change of $0.3 \mathrm{mg} /$ $\mathrm{dL}$ might need to be reconsidered. In addition, it should be kept in mind that the average laboratory measurement error in serum creatinine among hospitals is approximately $0.20 \pm$ $0.09 \mathrm{mg} / \mathrm{dL}$ [9].

It is also noteworthy that a poor prognosis was reported not only in the decreased kidney function group but also in the improved kidney function group with serum creatinine decline of $>0.05 \mathrm{mg} / \mathrm{dL}$; Kaplan-Meier survival analysis revealed that the stable kidney function group with $\Delta \mathrm{sCr}$ between $-0.05 \mathrm{mg} / \mathrm{dL}$ and $+0.05 \mathrm{mg} / \mathrm{dL}$ had the highest survival rate [4]. In addition to kidney function, changes in a patient's fluid balance and intravascular volume status might affect short-term (within 24 hours) changes in creatinine level. The improved kidney function group was comprised of patients who had reversible renal impairment at the time of admission, patients who were dehydrated upon admission, and patients who required fluid resuscitation due to severe septic conditions. Moreover, serum creatinine fluctuations as a result of impaired renal autoregulation can explain the survival difference to some extent. Previous clinical studies have reported a higher mortality in patients with greater kidney function variability in both outpatient [10] and inpatient [11] settings. Kao et al. [11] investigated the intraindividual variability of inpatient serum creatinine in 6,011 participants and found that creatinine change during hospitalization was significantly associated with in-hospital mortality. They proposed a threshold of $25 \mu \mathrm{mol} / \mathrm{L}(0.28 \mathrm{mg} / \mathrm{dL})$ for the creatinine change to predict a significantly higher mortality compared with those patients who had stable kidney function.

Several limitations should be considered when interpreting the results of Alfano et al. [4]: 1) their investigation was a retrospective study that selected patients with multiple creatinine measurements obtained within 24 hours; 2) the time between measurements varied within the 24-hour range; and 3 ) it is unclear whether the results are specific to non-ICU patients with COVID-19 or are relevant to a broader population, such as all critically ill patients with COVID-19 or patients with renal dysfunction associated with other diseases.

In conclusion, this timely report by Alfano et al. [4] sheds light on a small, potentially overlooked change in serum creatinine during the early stage of admission in patients with COVID-19. Future studies must validate the threshold that indicates significant creatinine change and the optimal measurement interval and also provide novel insights on the characteristics of COVID-19-associated AKI.

\section{Conflicts of interest}

All authors have no conflicts of interest to declare.

\section{ORCID}

Yohei Komaru, https://orcid.org/0000-0002-7970-5518

Kent Doi, https://orcid.org/0000-0002-0115-1439 


\section{References}

1. Chen YT, Shao SC, Hsu CK, Wu IW, Hung MJ, Chen YC. Incidence of acute kidney injury in COVID-19 infection: a systematic review and meta-analysis. Crit Care 2020;24:346.

2. Hirsch JS, Ng JH, Ross DW, et al. Acute kidney injury in patients hospitalized with COVID-19. Kidney Int 2020;98:209-218.

3. Gupta A, Madhavan MV, Sehgal K, et al. Extrapulmonary manifestations of COVID-19. Nat Med 2020;26:1017-1032.

4. Alfano G, Ferrari A, Fontana F, et al. Twenty-four-hour serum creatinine variation is associated with poor outcome in the novel coronavirus disease 2019 (COVID-19) patients. Kidney Res Clin Pract 2021;40:231-240.

5. Portolés J, Marques M, López-Sánchez P, et al. Chronic kidney disease and acute kidney injury in the COVID-19 Spanish outbreak. Nephrol Dial Transplant 2020;35:1353-1361.

6. Chan L, Chaudhary K, Saha A, et al. AKI in hospitalized patients with COVID-19. J Am Soc Nephrol 2021;32:151-160.
7. Lassnigg A, Schmidlin D, Mouhieddine M, et al. Minimal changes of serum creatinine predict prognosis in patients after cardiothoracic surgery: a prospective cohort study. J Am Soc Nephrol 2004;15:1597-1605.

8. Chertow GM, Burdick E, Honour M, Bonventre JV, Bates DW. Acute kidney injury, mortality, length of stay, and costs in hospitalized patients. J Am Soc Nephrol 2005;16:3365-3370.

9. Lee E, Collier CP, White CA. Interlaboratory Variability in Plasma Creatinine Measurement and the Relation with Estimated Glomerular Filtration Rate and Chronic Kidney Disease Diagnosis. Clin J Am Soc Nephrol 2017;12:29-37.

10. Al-Aly Z, Balasubramanian S, McDonald JR, Scherrer JF, O'Hare AM. Greater variability in kidney function is associated with an increased risk of death. Kidney Int 2012;82:1208-1214.

11. Kao SS, Kim SW, Horwood CM, Hakendorf P, Li JY, Thompson $\mathrm{CH}$. Variability in inpatient serum creatinine: its impact upon short- and long-term mortality. QJM 2015;108:781-787. 